\title{
Postnatal manipulation of Pax6 dosage reverses congenital tissue malformation defects
}

\author{
Cheryl Y. Gregory-Evans, ${ }^{1}$ Xia Wang, ${ }^{1}$ Kishor M. Wasan, ${ }^{2}$ Jinying Zhao, ${ }^{2}$ \\ Andrew L. Metcalfe, ${ }^{1}$ and Kevin Gregory-Evans ${ }^{1}$
}

1Department of Ophthalmology and 2Faculty of Pharmaceutical Sciences, University of British Columbia, Vancouver, British Columbia, Canada.

\begin{abstract}
Aniridia is a congenital and progressive panocular condition with poor visual prognosis that is associated with brain, olfactory, and pancreatic abnormalities. Development of aniridia is linked with nonsense mutations that result in paired box 6 (PAX6) haploinsufficiency. Here, we used a mouse model of aniridia to test the hypothesis that manipulation of Pax 6 dosage through a mutation-independent nonsense mutation suppression strategy would limit progressive, postnatal damage in the eye. We focused on the nonsense suppression drugs 3-[5-(2-fluorophenyl)-1,2,4-oxadiazol-3-yl]benzoic acid (ataluren) and gentamicin. Remarkably, we demonstrated that nonsense suppression not only inhibited disease progression but also stably reversed corneal, lens, and retinal malformation defects and restored electrical and behavioral responses of the retina. The most successful results were achieved through topical application of the drug formulation START ( $0.9 \%$ sodium chloride, $1 \%$ Tween $80,1 \%$ powdered ataluren, $1 \%$ carboxymethylcellulose), which was designed to enhance particle dispersion and to increase suspension viscosity. These observations suggest that the eye retains marked developmental plasticity into the postnatal period and remains sensitive to molecular remodeling. Furthermore, these data indicate that other neurological developmental anomalies associated with dosage-sensitive genetic mutations may be reversible through nonsense suppression therapeutics.
\end{abstract}

\section{Introduction}

The highly conserved paired box 6 (PAX6) transcription factor is pivotal to embryonic development and maintenance in the eye, brain, olfactory system, and pancreas (1). Genetic defects leading to haploinsufficiency of PAX6 cause congenital aniridia (2), a progressive panocular condition characterized by absence of iris tissue, corneal opacity, glaucoma, cataract, and foveal hypoplasia, which is also associated with brain, olfactory, and pancreatic abnormalities (3). While the genetic basis of congenital aniridia has been known for 2 decades, this has yet to be translated into preventative or corrective PAX6 treatment strategies, mainly because of PAX 6 allelic heterogeneity, since more than 600 different mutations are known (4). Premature stop codons (PTCs) caused by nonsense mutations, splice-site mutations, and frameshift mutations account for $72 \%$ of all PAX6 disease-associated mutations (4); however, approximately $50 \%$ of all mutations are in-frame nonsense mutations. Therefore, we reasoned that a mutation-independent nonsense mutation suppression approach for in-frame PTCs (4) could be relevant for many patients if this could be achieved through a postnatal strategy. In this approach, during mRNA translation, a near-cognate aminoacyl tRNA is inserted into the polypeptide. Thus, as long as the PTC is not in a critical position for protein activity, then a functional protein would be produced with the potential to provide therapeutic benefit (5).

We and others have previously undertaken proof-of-concept experiments showing that aminoglycosides promote readthrough of PTCs (6-9); however, they lack potency and are often associated with adverse systemic effects (10). Recently, nonsense-suppressing small molecules with limited toxic effects have been described (11). Therefore, in this study we tested a small molecule postnatal nonsense suppression strategy on the malformation defects exhibited by the Pax6-deficient mouse model of aniridia ( $\left.\mathrm{Pax}^{\mathrm{Sey+}+-}\right)$.

Conflict of interest: The authors have declared that no conflict of interest exists. Citation for this article: J Clin Invest. 2014;124(1):111-116. doi:10.1172/JCI70462.

\section{Results and Discussion}

Postnatal systemic nonsense suppression. As we had previously tested aminoglycosides in rat and zebrafish nonsense mutations models $(8,9)$, we chose gentamicin for preliminary tests in vivo. We also selected 3-[5-(2-fluorophenyl)-1,2,4-oxadiazol-3-yl]benzoic acid (ataluren) for nonsense suppression, because it is known to be the least toxic drug in humans (12). For in vivo studies, we used the semidominant small eye ( $\left.\mathrm{Pax} 6^{\mathrm{Sey+}+-}\right)$ model of aniridia, which has a naturally occurring Gly194X stop codon mutation (UGA) in the mouse Pax6 gene (13). Our initial therapeutic paradigm was daily subcutaneous injections of $30 \mu \mathrm{g} / \mathrm{g}$ ataluren or $6.25 \mu \mathrm{g} / \mathrm{g}$ gentamicin from P4 to P14 in $\mathrm{Pax}^{\mathrm{Sey+} /-}$ mice. In mutant eyes, prior to treatment at P4, the cornea was thickened, there was a lenticular stalk in which the underdeveloped lens was attached to the cornea, and the retina was thickened and abnormally infolded at the ciliary margin (Figure 1A). Without treatment, at P14 the Pax $6^{\mathrm{Sey+}+}$ globe was further distorted, progressive infolding was seen in the mutant retina, and the lens was abnormally small. However, ataluren treatment resulted in correction of retinal infolding and also resulted in an increase in lens size to $70 \%$ of that observed in wild-type eyes. Similarly, treatment with gentamicin, starting either prenatally at E12.5 or postnatally at P4 and continuing until P21, resulted in remarkable normalization of the eye malformation defects (Supplemental Figure 2; supplemental material available online with this article; doi:10.1172/JCI70462DS1). This demonstrated that tissue development controlled by Pax 6 expression is still sensitive to manipulation after birth. However, observations in the anterior segment of the eye showed that the corneal stroma in treated animals was abnormally thickened (Figure 2A). Detailed analysis showed that at P14 the thickness of the corneal epithelium in untreated eyes was $9 \pm 2 \mu \mathrm{m}(n=6)$, in treated eyes was $10 \pm 3 \mu \mathrm{m}$ $(n=6)$, and in wild-type eyes measured $38 \pm 2 \mu \mathrm{m}$ (Figure $2 \mathrm{~B}$ ). Continued treatment with ataluren to P60 did not significantly 


\section{brief report}

A

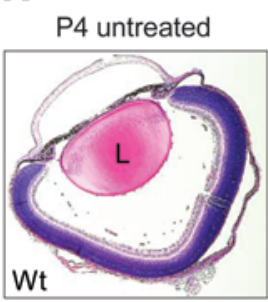

P14 untreated
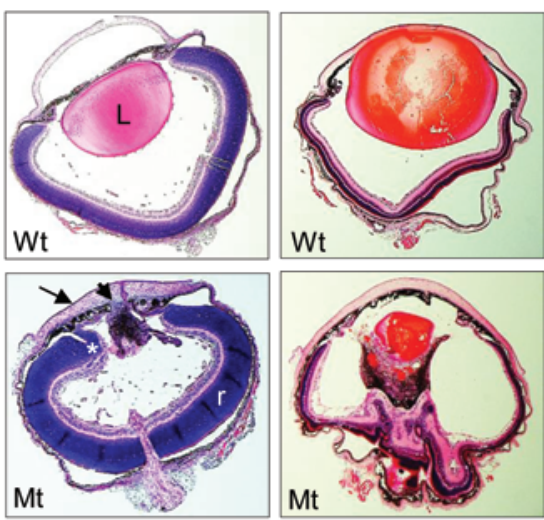

Mt

\section{C}

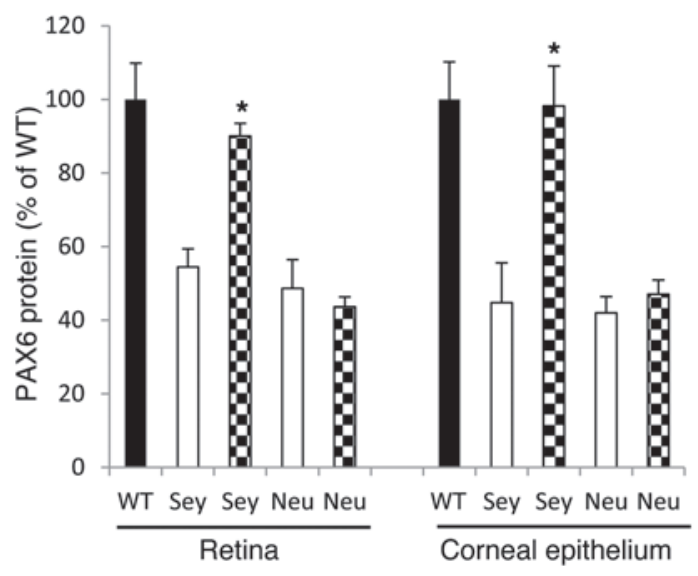

P4-P14 systemic Ataluren
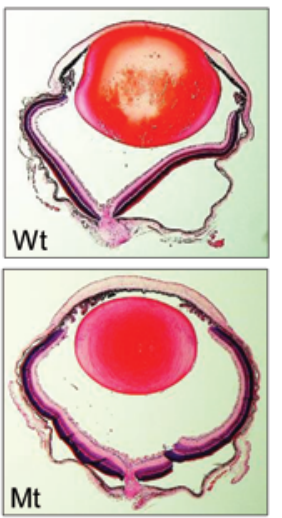

Mt
B
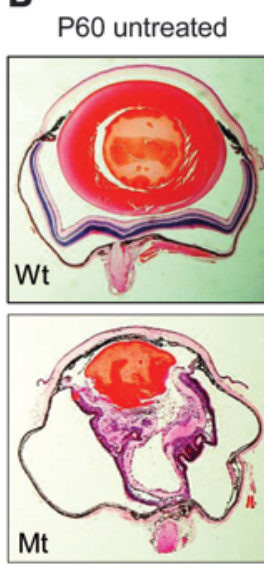

D

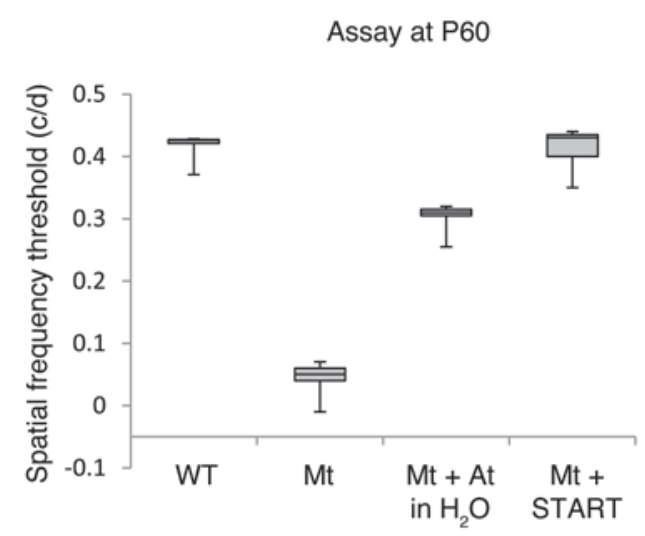

C
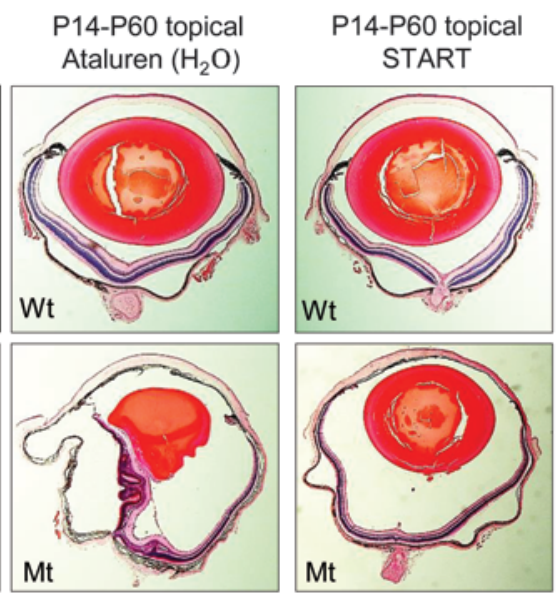

Wt

P14-P60 topical START
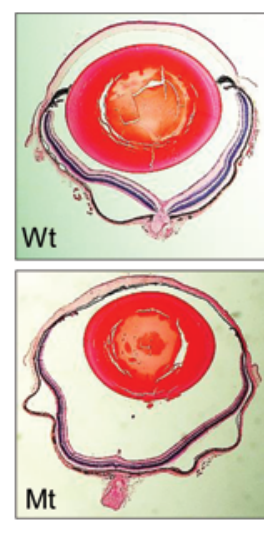

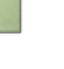


A Anterior segment P14

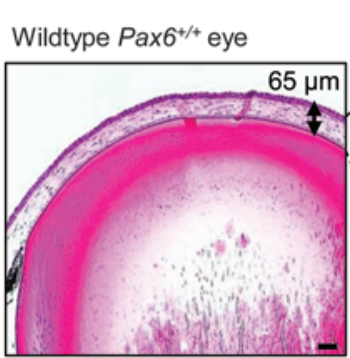

Pax6 ${ }^{\text {Seyt// }}$ treated eye

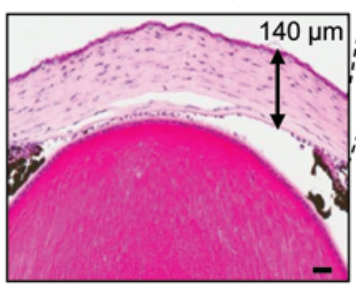

B Cornea-systemic
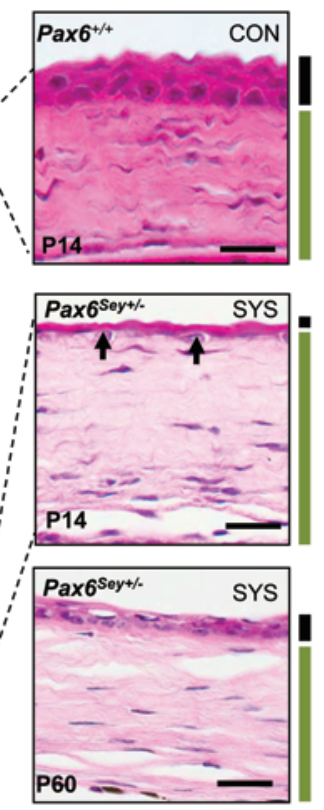

\section{Cornea-topical}
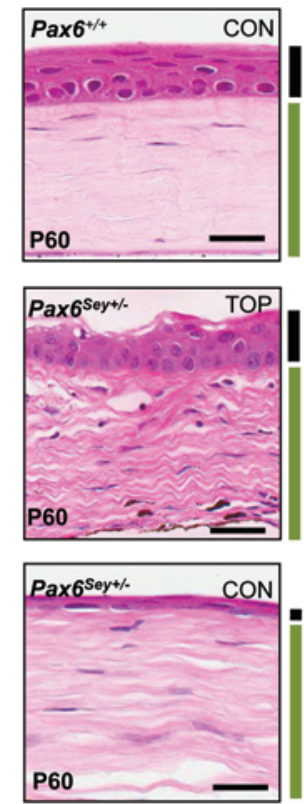

D Epithelial corneal stratification

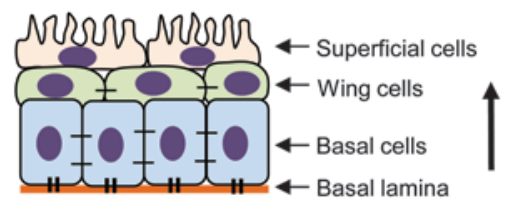

E Retina at P14

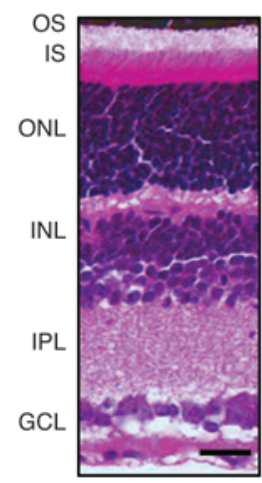

$P^{2 a x} 6^{+/+}$

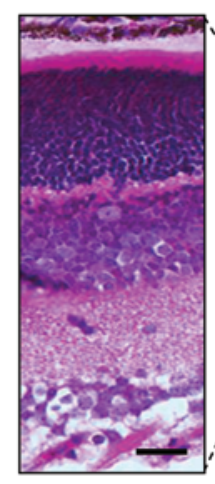

Pax6 ${ }^{\text {Sey+/ }}$ treated

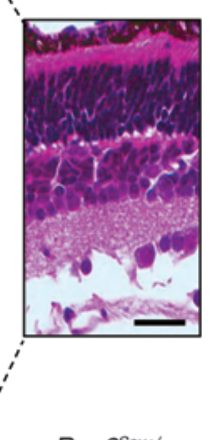

Pax6 ${ }^{\text {Sey+1- }}$ untreated

\section{Figure 2}

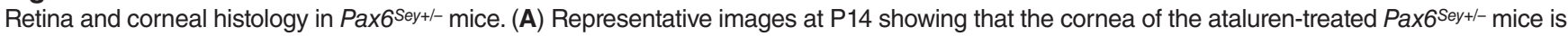
thicker than wild-type $\left(\mathrm{Pax}^{+/+}\right)$cornea $(140 \mu \mathrm{m}$ versus $65 \mu \mathrm{m}, n=6)$. Scale bar: $100 \mu \mathrm{m}$. (B) Higher magnification of corneal tissue at P14 and P60. The stroma (green bar) is thicker and the epithelium (black bar) is abnormally thin with systemic (SYS) treatment. Arrows indicate basal cells. Scale bar: $10 \mu \mathrm{m}$. (C) Topical (TOP) treatment with the START formulation at P60 in Pax6Sey+/- eyes. Untreated Pax6Sey+/- control (CON) corneal epithelium remains thin at P60. Scale bar: $10 \mu \mathrm{m}$. (D) Epithelial stratification of the cornea. (E) Retinal sections from wild-type, systemically treated $P a x 6^{\text {Sey+l- }}$ mice and untreated mice, showing the photoreceptor inner segments (IS) and outer segments (OS) are shorter in the treated mice ( $n=6)$. The nuclei in the outer nuclear layer (ONL) are more densely packed in the treated mice compared with those in the wild-type mice. Scale bar: 50 um. All the retinal layers in the untreated mice are thinner than normal. INL, inner nuclear layer; IPL, inner plexiform layer; GCL, ganglion cell layer.

would increase drug concentration in the eye while further limiting any systemic toxicity (12). A $1 \%$ aqueous ataluren suspension was delivered topically to the eye (50-100 $\mu$ g per eye) twice a day from P14 (when the eyes open) to P60. This resulted in only partial histological rescue of the retina and lens defects (Figure 1B). Also, we noted that the ataluren suspension caused marked ocular irritation in treated animals immediately upon drug administration. We therefore tested a number of reformulations of the drug suspension to improve particle dispersion properties (Supplemental Figure 1) and to increase the suspension viscosity so that the drug would remain in contact with the ocular surface for longer. The final novel formulation, referred to as START ( $0.9 \%$ sodium chloride, $1 \%$ Tween $80,1 \%$ powdered ataluren, $1 \%$ carboxymethylcellulose), abolished the irritation response seen with other formulations. The lens and retinal defects had been reversed in the treated $\mathrm{Pax}^{\mathrm{Sey+}+-}$ eyes (Figure 1B) to more closely resemble wild-type eyes than was achievable with either topical $1 \%$ aqueous ataluren or with systemic delivery of the drug. In addition, ocular histology showed that the corneal epithelium measured $40 \pm 3 \mu \mathrm{m}(n=6)$ in thickness in eyes treated with START therapy (Figure 2C), demonstrating that topical treatment had rescued the corneal deficit to wild-type levels. To confirm directly that START therapy in vivo lead to an increase in PAX6 protein, ELISA analysis of treated $\mathrm{Pax} 6^{\mathrm{Sey+} /-}$ retinal and corneal epithelium protein lysates revealed that PAX6 protein levels increased to $90 \% \pm 5 \%(n=6)$ of those of wild-type mice
(Figure 1C). For comparison, a mouse model with a Pax6 splicesite mutation (Pax6 $6^{\text {Sey-1Neu }}$ mice) (13) did not respond to START therapy, indicating specificity for nonsense mutations.

Topical nonsense suppression improves functional deficits of the retina. ERG testing showed that both topical formulations resulted in improved responses to light stimuli (Figure 3A, traces 4 and 5). However, the scotopic and photopic b-wave responses for topical ataluren in $\mathrm{H}_{2} \mathrm{O}$ were smaller than those in wild-type animals (35\% and $29 \%$, respectively, $n=5$ ), which were similar to systemic treatment responses (Figure 3B). One-way ANOVA with Tukey posthoc comparisons between treatment groups showed no statistically significant difference in the responses between systemic and topical ataluren in $\mathrm{H}_{2} \mathrm{O}(P=0.994)$. However, the responses for START therapy showed a significant benefit over other treatments $(P<0.01)$. Additionally, the scotopic b-wave amplitudes in STARTtreated animals were not significantly different from those of wildtype controls $(P=0.78)$.

To further evaluate the functional effects of treatment, we assessed the optokinetic tracking response, a behavioral response mediated through retina-brain circuitry (15) that approximates a measurement of visual acuity. Under optokinetic tracking test conditions, $\mathrm{Pax} 6^{\mathrm{Sey+}+-}$ mutants demonstrated limited tracking responses (0.04 cycles/degree [c/d]) (Figure 1D) when compared with those of wild-type mice $(0.42 \mathrm{c} / \mathrm{d})$. Mice treated topically with aqueous ataluren had an improved spatial frequency threshold $(0.3 \mathrm{c} / \mathrm{d})$. However, mice treated with the 

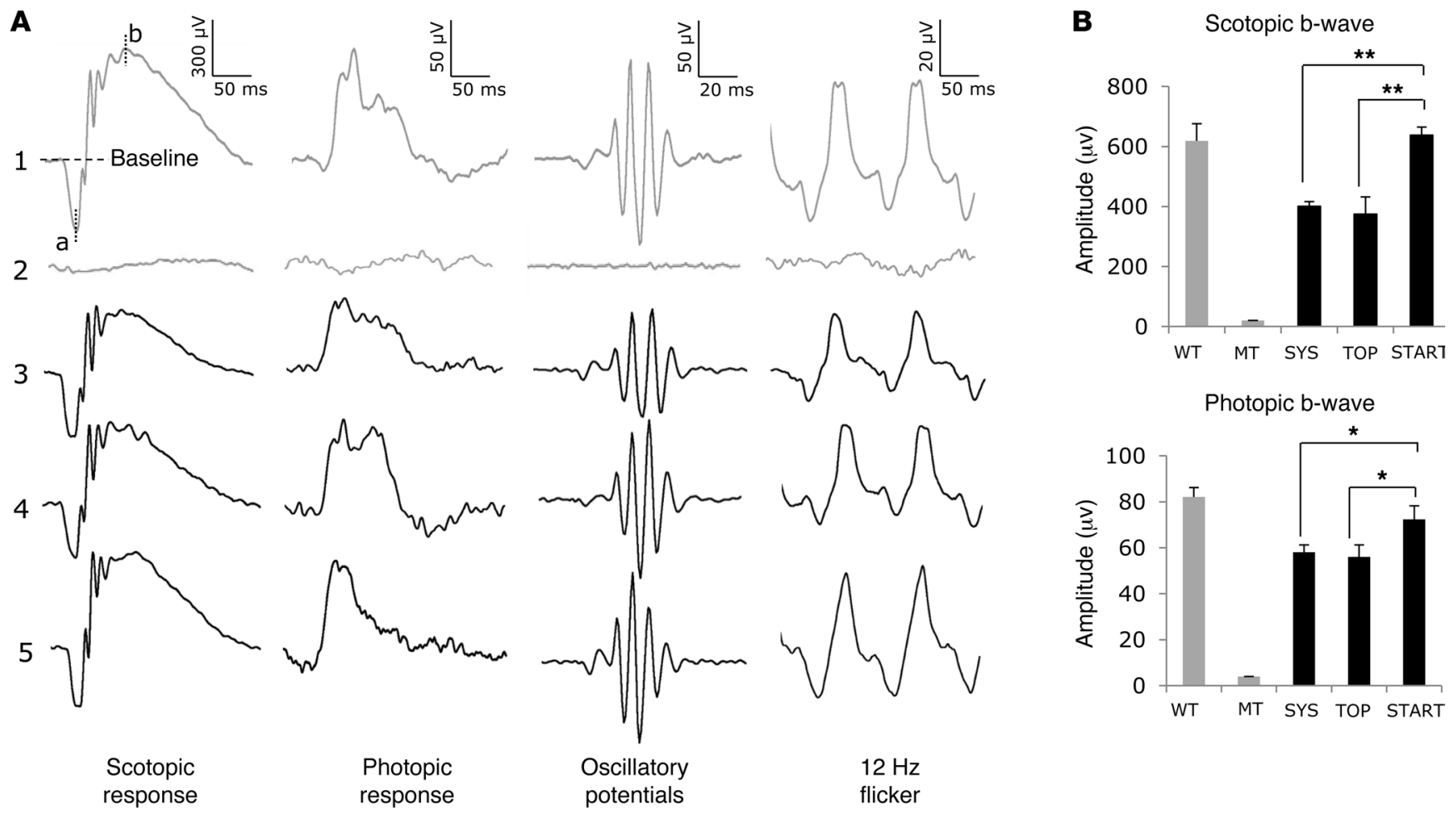

\section{Figure 3}

Electroretinographic analyses in response to light stimulation. (A) Restoration of light sensitivity in Pax6Sey+/- eyes measured at P60. Trace 1 shows wild-type mice with systemic ataluren. Trace 2 shows untreated Pax $6^{\text {Sey+l- }}$ mouse responses. Trace 3 shows $P a x 6^{\text {Sey+l- }}$ mice with systemic ataluren. Trace 4 shows Pax6 $6^{\text {Sey+l- }}$ mice with topical $1 \%$ ataluren suspension. Trace 5 shows Pax6 ${ }^{\text {Sey+l- }}$ mice with topical START formulation. a, a-wave maximum from baseline; b, b-wave maximum from baseline. (B) Quantification of scotopic and photopic b-wave responses $(n=6)$. Significance determined by 1 -way ANOVA with Tukey post-hoc tests ${ }^{\star} P<0.05 ;{ }^{*} P<0.01$.

START formulation showed higher spatial frequency thresholds $(0.42 \mathrm{c} / \mathrm{d})$, and these were not significantly different from responses seen in wild-type animals $(P=0.42)$.

Here, we demonstrated that, in the haploinsufficient postnatal mouse eye, manipulation of Pax 6 dosage stably reversed the congenital ocular malformations and, importantly, that this was best achieved through topical administration using the START formulation. Based on our observations, we propose that abnormally developed ocular tissue is still remarkably sensitive to molecular remodeling postnatally. In mammals, although most tissue morphogenesis is completed by birth, it has already been found that this is not the case in the CNS. Both the brain and eye continue to adapt after birth, with significant remodeling of new synaptic connections (16). Our data suggest that this postnatal plasticity might be sufficiently versatile to also allow for reversal of abnormal tissue modeling if the underlying molecular defect is corrected. The mechanism by which this remodeling occurs warrants further investigation.

Another key finding in this study was that extensive remodeling could be achieved in vivo in the retina and corneal epithelium with START therapy that correlated directly with increasing PAX6 protein expression to approximately $90 \%$ of wild-type levels. Based on previous studies $(11,12)$, this suggests that ataluren was able to suppress the nonsense mutation, allowing full-length PAX6 protein to be synthesized. Recently, the effectiveness of ataluren as a nonsense suppression agent has been questioned (17). Despite this, several in vivo studies (12, 18), including this one, show beneficial efficacy of ataluren as a treatment strategy. Further research is needed to fully define the exact mechanism of action of this drug.

The effects of varying Pax 6 gene dosage have been investigated in haploinsufficient and overexpression models of ocular malformation (19). However, the minimum Pax6 dosage that is required for normal tissue development has yet to be determined, but it is likely to vary in different tissues and at different times. Other genes associated with abnormal ocular development, such as Pitx2, Foxc1, Foxc2, and Foxe3, are also dosage-sensitive (20-22). Manipulation of these genes after birth may also lead to corrective tissue remodeling, suggesting a common therapeutic paradigm that could be applicable to many congenital eye defects. In addition, nonsense suppression therapy is also emerging as a therapy outside the eye. For example, in cystic fibrosis (7) and Duchenne muscular dystrophy mouse models (18), ataluren treatment lead to an approximately $25 \%$ increase in protein production, and this correlated with a similar improvement in functional studies. Thus, a moderate increase in gene expression is able to have a beneficial effect, but presumably the levels would need to be closer to $50 \%$ in the affected tissues for full rescue.

In conclusion, we have uncovered a viable therapeutic option for children with PAX6 nonsense mutations that reverses a debilitating eye malformation defect. In addition, it is also advantageous that the most benefit appears linked to topical application, 
Table 1

ERG data for different treatment regimens

\begin{tabular}{|c|c|c|c|c|}
\hline Mice and treatment & $\begin{array}{l}\text { Scotopic a-wave } \\
(\mu V \pm \text { SEM) }\end{array}$ & $\begin{array}{l}\text { Scotopic b-wave } \\
(\mu V \pm \text { SEM) }\end{array}$ & $\begin{array}{l}\text { Photopic b-wave } \\
(\mu V \pm \text { SEM) }\end{array}$ & $\begin{array}{l}\text { 12-Hz flicker b-wave } \\
\quad(\mu \mathrm{V} \pm \mathrm{SEM})\end{array}$ \\
\hline $\begin{array}{l}\text { Wild-type mice untreated } \\
\text { at P60 }(n=6)\end{array}$ & $-274.2 \pm 18.7$ & $619.1 \pm 57.1$ & $82.1 \pm 4.1$ & $42.1 \pm 4.9$ \\
\hline $\begin{array}{l}\text { Pax6 } 6^{\text {Sey+/- }} \text { mice untreated } \\
\text { at P60 }(n=6)\end{array}$ & Undetectable & Undetectable & Undetectable & Undetectable \\
\hline $\begin{array}{l}\text { Pax6 } 6^{\text {Sey+- }} \text { mice with systemic therapy } \\
\text { to P60 }(n=6)\end{array}$ & $-200.7 \pm 26.3$ & $400.8 \pm 15.3$ & $58.1 \pm 3.1$ & $30.6 \pm 3.3$ \\
\hline $\begin{array}{l}\text { Pax6 }{ }^{\text {Sey+ }+-} \text { mice with systemic therapy } \\
\text { to P21, drug withdrawal to P60 }(n=6)\end{array}$ & $-210.0 \pm 18.9$ & $439.4 \pm 12.1$ & $61.4 \pm 8.82$ & $29.7 \pm 3.91$ \\
\hline $\begin{array}{l}\text { Pax6 }{ }^{\text {Sey+ }+-} \text { mice with topical } 1 \% \text { ataluren } \\
\text { in } \mathrm{H}_{2} \mathrm{O} \text { to } \mathrm{P} 60(n=6)\end{array}$ & $-161.7 \pm 27.8$ & $374.5 \pm 57.4$ & $55.9 \pm 5.2$ & $32.1 \pm 3.7$ \\
\hline $\begin{array}{l}\text { Pax6 } \text { Sey+1- mice with topical START } \text { formulation to P60 }(n=6)\end{array}$ & $-215.8 \pm 20.3$ & $638.0 \pm 26.9$ & $72.4 \pm 5.8$ & $38.1 \pm 2.1$ \\
\hline
\end{tabular}

since this will improve patient compliance and reduce the risk of systemic adverse effects to a minimum. Based on this study in a directly relevant animal model, we would advocate the initiation of clinical trials for a nonsense suppression paradigm in aniridia.

\section{Methods}

Animals and drug administration. Both Pax6 mouse alleles (Pax6 $6^{\text {Sey+--; }}$ a gift of Veronica van Heyningen, MRC Human Genetics Unit, Edinburgh, United Kingdom, and Pax6 $6^{\mathrm{Sey}-1 \mathrm{Neu} ;}$ a gift of Elizabeth Simpson, University of British Columbia) were maintained on the C57BL/6 background. Pax6 mutant offspring were identified by genotyping (details in Supplemental Methods). Daily subcutaneous injections of $30 \mu \mathrm{g} / \mathrm{g}$ body weight of an aqueous suspension of ataluren (Selleckchem) started at P4 and continued until P14. For gentamicin studies, subcutaneous injections $(6.25 \mu \mathrm{g} / \mathrm{g}$; Sigma-Aldrich) were carried out from P4 to P21 or began in time-mated pregnant mice from E12.5 to P21. For topical administration, drops were instilled into the eyes twice daily beginning at P14 until analysis at P60 using 2 different formulations: a $1 \%$ ataluren aqueous suspension or the START formulation. START drug formulation details are in the Supplemental Methods and Supplemental Table 1.

Histology. Eyes were fixed in 50\% Karnovsky fixative. Paraffin-embedded eye sections were stained with $\mathrm{H} \& \mathrm{E}$ and photographed using either a Leica stereomicroscope or an Aperio ScanScope digital scanning system. Lens size was estimated using the Image J program.

Pax6 ELISA. Corneal epithelium was dissected off the ocular surface into $100 \mu \mathrm{l}$ of $1 \mathrm{X}$ PBS. After removing the anterior segment and lens, retinal tissue was dissected out of the eye cup into $100 \mu$ l of $1 \mathrm{X}$ PBS. Both tissue samples were then homogenized, followed by 2 freeze-thaw cycles. Homogenates were centrifuged for 5 minutes at $5,000 \mathrm{~g}$, and the supernatant was immediately assayed with a mouse PAX6 ELISA Kit (MyBioSource) according to manufacturer's instructions.
ERG. Responses of the retina to light flashes were recorded as previously described (11) using an Espion E2 system with a ColorDome mini-Ganzfeld stimulator (Diagnosys LLC). Dark-adapted responses were recorded at P60 by averaging 15 responses at a stimulus intensity of $3.16 \mathrm{c} / \mathrm{d} \mathrm{s} / \mathrm{m}^{2}$. Lightadapted cone responses were carried out in $30 \mathrm{c} / \mathrm{d} / \mathrm{m}^{2}$ background light.

Optokinetic tracking. We assessed behavioral responses using optokinetic tracking (15) using rodent-specific OptoMotry equipment (Cerebral Mechanics). Box-and-whisker plots were prepared showing the 5\% and $95 \%$ quantiles (whiskers), $25 \%$ and $75 \%$ quartiles (box), and the median marked by a horizontal line.

Statistics. Analyses were performed with GraphPad Prism 5.0. For parameter comparisons between groups, an unpaired 2-tailed Student's $t$ test or 1-way ANOVA followed by Tukey multiple comparison test were used when appropriate. $P$ values of less than 0.05 were considered significant. Results are reported as mean \pm SEM.

Study approval. Research was carried in accordance with the Canadian Council on Animal Care, with the approval of the Animal Care Committee at the University of British Columbia (study no. A09-0941).

\section{Acknowledgments}

This work was supported by the Sharon Stewart Aniridia Trust (no. F12-02746) and the Canadian Institutes of Health Research (no. 222728).

Received for publication April 12, 2013, and accepted in revised form September 27, 2013.

Address correspondence to: Cheryl Y. Gregory-Evans, Department of Ophthalmology, University of British Columbia, 2250 Willow Street, Vancouver, British Columbia, V5Z 3N9, Canada. Phone: 604.875.5529; Fax: 604.875.4663; E-mail: cge30@mail.ubc.ca.
1. Simpson TI, Price DJ. Pax6: a pleiotropic player in development. Bioessays. 2002;24(11):1041-1051.

2. Ton CC, et al. Positional cloning and characterization of a paired box- and homeobox-containing gene from the aniridia region. Cell. 1991;67(6):1059-1074.

3. Sisodiya SM, et al. PAX6 haploinsufficiency causes cerebral malformation and olfactory dysfunction in humans. Nat Genet. 2001;28(3):214-216.

4. Tzoulaki I, White IM, Hanson IM. PAX6 mutations: genotype-phenotype correlations. $B M C$
Genet. 2005;6(1):27.

5. Keeling KM, Wang D, Conard SE, Bedwell DM. Suppression of premature termination codons as a therapeutic approach. Crit Rev Biochem Mol Biol. 2012;47(5):444-463.

6. Barton-Davis ER, Cordier L, Shoturma DI, Leland SE, Sweeney HL. Aminoglycoside antibiotics restore dystrophin function to skeletal muscles of mdx mice. J Clin Invest. 1999;104(4):375-381.

7. Du M, Liu X, Welch EM, Hirawat S, Peltz SW, Bed- well DM. PTC124 is an orally bioavailable compound that promotes suppression of the human CFTR-G542X nonsense allele in a CF mouse model. Proc Natl Acad Sci US A. 2008;105(6):2064-2069.

8. Moosajee M, Gregory-Evans K, Ellis CD, Seabra MC, Gregory-Evans CY. Translational bypass of nonsense mutations in zebrafish rep1, pax2.1 and lamb1 highlights a viable therapeutic option for untreatable genetic eye disease. Hum Mol Genet. 2008;17(24):3987-4000. 
9. Guerin K, et al. Systemic aminoglycoside treatment in rodent models of retinitis pigmentosa. Exp Eye Res. 2008;87(3):197-201.

10. Wilschanski M, et al. Gentamicin-induced correction of CFTR function in patients with cystic fibrosis and CFTR stop mutations. $N$ Engl J Med. 2003;349(15):1433-1441.

11. Welch EM, et al. PTC124 targets genetic disorders caused by nonsense mutations. Nature. 2007;447(7140):87-91.

12. Wilschanski M, et al. Chronic Ataluren (PTC124) treatment of nonsense mutation cystic fibrosis. Eur Respir J. 2011;38(1):59-69.

13. Hill R, et al. Mouse Small eye results from mutations in a paired-like homeobox-containing gene. Nature. 1991;354(6354):522-525.

14. Kroeber M, et al. Reduced expression of Pax6 in lens and cornea of mutant mice leads to failure of chamber angle development and juvenile glaucoma. Hum Mol Genet. 2010;19(17):3332-3342.

15. Douglas RM, Alam NM, Silver BD, McGill TJ, Tschetter WW, Prusky GT. Independent visual threshold measurements in the two eyes of freely moving rats and mice using a virtual-reality optokinetic system. Vis Neurosci. 2005;22(5):677-684.

16. Bourne JA. Unravelling the development of the visual cortex: implications for plasticity and repair. J Anat. 2010;217(4):449-468.

17. McElroy SP, et al. A lack of premature termination codon read-through efficacy of PTC124 (Ataluren) in a diverse array of reporter assays. PLoS Biol. 2013;11(6):e1001593.

18. Kayali R, Ku JM, Khitrov G, Jung ME, Prikhodko O, Bertoni C. Read-through compound 13 restores dys- trophin expression and improves muscle function in the mdx mouse model for Duchenne muscular dystrophy. Hum Mol Genet. 2012;21(18):4007-4020.

19. Schedl A, et al. Influence of PAX6 gene dosage on development: Overexpression causes severe eye abnormalities. Cell. 1996;86(1):71-82.

20. Gage PJ, Suh H, Camper SA. Dosage requirements of Pitx2 for development of multiple organs. Development. 1999;126(20):4643-4651.

21. Smith RS, et al. Haploinsufficiency of the transcription factors FOXC1 an FOXC2 results in aberrant ocular development. Hum Mol Genet. 2000;9(7):1021-1032.

22. Ormestad M, Blixt A, Churchill A, Martinsson T, Enerback S, Carlsson P. Foxe3 haploinsufficiency in mice: a model for Peter's anomaly. Invest Ophthalmol Vis Sci. 2002;43(5):1350-1357. 